Grasas y Aceites

\title{
Effect of type and level of dietary fibre supplements in rats
}

\author{
By Mona M. Rashad and Sorial A. Moharib
}

\author{
Biochemistry Department, National Research Centre, Tahrir St., Dokki, Cairo, Egypt.
}

\section{RESUMEN}

Efecto del tipo y nivel de fibra dietética suplementada en ratas.

Estudios clínicos y experimentales han indicado que nuevas fuentes de fibras dietéticas son potencialmente hipolipídicas. En ul presente estudio nabo, remolacha, col y fenogreco verde. En lo presente estudio nabo, remolacha, col y fenogreco verde fueron utilizados como nuevas fuentes naturales de fibra dietética para examinar sus efectos en parámetros nutricionales y en e metabolismo lípidico de ratas alimentadas durante 8 semanas comparando con una dieta control (control-libre de fibra). El análisis químico de las 4 plantas revelo que hay diferencias entre las proporciones de polisacáridos no amiláceos insolubles y solubles (NSP) y sus constituyentes monómeros. Los niveles mas altos de ácidos urónicos se presentan en la fibra soluble (NSP) que en la insoluble, para todas las fuentes de fibra. Se observa una disminución altamente significativa en la ganancia de peso y en la ingesta de alimentos para las ratas alimentadas, con las 4 dietas experimentales, después de 8 semanas, comparado con la dieta control. Las ratas alimentadas con la dieta de nabo muestran una disminución altamente significativa en todos los parámetros nutricionales durante el periodo de alimentación. Las ratas alimentadas con una dieta suplementada con fibra de remolacha tienen una disminución altamente significativo en el valor de ganancia de una disminución alimento y peso, ingesta de alime de alimentación. Las dietas con contenido en fibra de remolacha, col y fenogreco verde tienen un efecto de disminución de los niveles en suero de lípido total, colesterol total, LDL-C (excepto remolacha) y triglicéridos sobre las ratas alimentadas con la dieta control. La dieta suplementada con fibra de nabo tiene solo disminuye los triglicéridos en suero durante el periodo de alimentación. Los niveles de lípido total y colesterol total en hígado fueron significativamente más bajos cuando las ratas se alimentan con dietas de col y fenugreco verde. Existe una disminución altamente significativa en el nivel de triglicéridos en el hígado de ratas alimentadas con dieta suplementada con fibra de nabo comparando con el periodo experimental (8 semanas).

PALABRAS-CLAVE: Fibra dietética - Hiperlipidemia - Hojas de plantas - NSP - Parámetros nutricionales -Rata.

\section{SUMMARY} rats.

Effect of type and level of dietary fibre supplements in

Both experimental and clinical studies have indicated that a novel source of dietary fibres is potentially hypolipidemic. In the present study, turnip, sugar beet, cabbage and fenugreek green leaves were used as natural new sources of dietary fibres, to eaves were used as natural new sources of dietary fibres, to . metabolism in rats fed for 8 weeks comparing with a control die ises of the 4 plants revealed that there are differences between the ratios of soluble and insoluble non - starch polysaccharides (NSP) and their monomers constituents. Higher levels of uronic acid were present in soluble fibre (NSP) in all fibre sources than that of insoluble one. Generally, highly significant decrease in the weight gain and food intake of the rats fed the 4 experimental diets after 8 weeks feeding comparing with those fed control diet. Rats fed turnip diet exhibited a highly significant decrease in all the nutritional parameters through the feeding period. Rats fed a diet supplemented with sugar beet fibre, have a highly significant decrease in the value of weight gain, food intake and Dapp over the feeding period. Sugar beet, cabbage and fenugreek green fibre-containing diets had lowering effect on serum total lipid, tota cholesterol, LDL-C (except sugar beet) and triglycerides levels than that of control diet fed rats. Supplemented the diet with turnip fibre had only lowering effect on serum triglycerides over the feeding period. Hepatic total lipid and total cholesterol levels were significantly lower when rats fed cabbage and fenugreek green fiber-containing diets. Highly significant decrease in the level of triglycerides was achieved in the liver of rats fed turnip fibre supplemented diet comparing with the experimental period (8 weeks).

KEY-WORDS: Dietary fibre -- Hyperlipidaemia - NSP - Nutritional parameters --Plant leaves - Rats.

\section{INTRODUCTION}

Foods are complex mixtures containing different relative molecular masses and concentrations of fermentable carbohydrates. The complexity and the variety of these components may allow maintenance of high fermentation activity throughout the large intestine, which can increase their beneficial effects on mineral absorption (Campbell et al., 1997 and Younes et al., 2001). Dietary fibre is used increasingly in human and animal nutrition (Guerin et al., 2001). Previous investigations demonstrated the potential effects of different sources of dietary fibres on nutritional parameters and growth rates (Johnson and Gee, 1986; Jwanny et al., 1996 and Moharib, 2000).

Dietary fibres have been the subject of numerous epidemiological studies, which for the most part have demonstrated an inverse relationship between high fibre content and diseases (cancer, coronary heart disease, cardiovascular disease, hypertension, atherosclerosis and hyperlipidaemia) (Onning and Asp, 1995, Birt et al., 1998 and Sapuntzakis et al., 2001). Other investigators (Jacobs, 1983; Nyman and Asp, 1985 and Buhman et al., 1998) provided evidence that fibre consumption results in protection against chemically induced large bowel microflora. Lajvardi et al. (1993) and Campbell et al. (1997) stated that the effects are highly dependent on the experimental conditions and type and levels of the fibre used. Several studies have shown the beneficial effect of supplement the rat diets with either soluble 
or insoluble dietary fibre fractions of plant leaves and vegetables or as a mixture of both. (Vahouny et al., 1987; Schneeman and Gallaher, 1993; Onning and Asp, 1995; Birt et al., 1998; Goel et al., 1999; Gallaher et al., 2000 and Moharib, 2000). They concluded that these fibres could be used as hypolipidemic and hypocholesterolemic agents.

The aim of the present study is directed to study the effect of feeding male albino rats different level and types of some Egyptian plant leaves dietary fibres on lipid metabolism. Also, their effects on nutritional parameters and growth rates were studied.

\section{MATERIALS AND METHODS}

\subsection{Materials}

Fresh leafy materials of sugar beet (Beta vulgaris), cabbage (Brassica oleracea), turnip (Brassica rapa) and Fenugreek green (Corchorus olitorius) were collected from an Egyptian local market. The plants were cut into pieces of $30 \mathrm{~mm}$ length, known weight of plant leaves were ground finally in a food grinder (mincer), squeezing them with a manual screw press through cloth for removing the liquid juice for protein production (LPC), (Rashad et al., 2000). The residues formed were collected and dried in a freeze dryer at $-60^{\circ} \mathrm{C}$, subsequently the dry residues were ground finally in a coffee grinder. The ground fibres were sifted though a 16 mesh sieve, packed in bags and stored at room temperature till used for chemical composition analysis.

\subsection{Diets}

The composition of the fibre-free diet (control) (Johnson and Gee 1986) and 4 experimental diets was shown in Table II. Each experimental diet supplemented with one of the 4 different sources of dryed, grounded and sifted plant leaves fibre.

\subsection{Animal}

One hundred-forty male albino rats (Rattus norvgicus), 6 weeks of age, weighing about $115 \mathrm{~g}$ were purchased from the Egyptian Organization for Biological products and Vaccines and fed with a commercial diet for a period of 2 weeks. The rats were then divided into five groups of 28 rats per each on the basis of their body weight and individually housed in wire screen cages. The first group was fed fibre-free control diet and the other groups received one of the four experimental diets. The rats had free access to food and tap water and the diets were maintained for a period of 8 weeks. The animal room was temperature controlled $\left(29 \pm 1^{\circ} \mathrm{C}\right)$ and had a 12-hour light-dark cycle. The experimental protocol was done according to the methods of Silva and Nicoli, (1985) and Jwanny et al., (1996).

\subsection{Analytical methods for dietary fibres}

Lipids were extracted with chloroform-methanol mixture $(2: 1 \mathrm{v} / \mathrm{v})$ according to the method of Folch et al., (1957). Starch and non-starch polysaccharides (NSP), either soluble or insoluble and cellulosic and non-cellulosic polysaccharides (NCP) were estimated chemically and enzymatically according to the method described by Englyst and Cummings, (1988). Qualitative and quantitative determination of NCP hydrolysates were done according to the method of Wilson, (1959). Neutral monosaccharides of each soluble and insoluble NSP were measured by the method of Jwanny and Hussein, (1976), using paper chromatographic technique. Uronic acids were measured by a colorimetric method (Englyst and Cummings, 1988).

\subsection{Nutritional and metabolic parameters}

During the feeding period (8 weeks), the rat faeces were collected daily and dried in an oven at $105^{\circ} \mathrm{C}$, weighed and tested for nitrogen content. Weight gain, food intake, protein efficiency ratio (PER), feed efficiency ratio (FER), apparent digestibility (Dapp) and food conversion efficiency (FCE) were calculated every 2 weeks of feeding according to the methods described by Silva and Nicoli, (1985) and Johnson and Gee, (1986). At the end of every two weeks, blood samples were draw from the retroorbital venous plexus with heparinized capillary tubes. Chemical analysis of the separated serum was done using Boehringer-Mannheim Kit, total lipids content using the method of Knight et al., (1972), total cholesterol by Trinder method (1969), high density lipoprotein cholesterol (HDL-C) and low density lipoprotein cholesterol (LDL-C) using Lopes-Virella et al., (1977) method and triglycerides according to Wahlefeld (1974) method. Weighed liver samples of the 7 individual rats from each group were taken after every two weeks of feeding and lipid extracted using Folch et al., (1957). The levels of total lipid, total cholesterol and triglycerides in the extracted liver were determined by the kit using the method mentioned above as in serum. Statistical analysis was performed by students t-test (Fisher, 1970).

\section{RESULTS}

\subsection{Composition of dietary fibres}

Chemical analysis of the 4 different plant fibres (Table I) showed that turnip fibres contained equal 
Table I

Chemical composition of dietary fibre components of non- starch polysaccharides (NSP) of different plant leaves

\begin{tabular}{|c|c|c|c|c|c|c|c|c|c|}
\hline \multirow{2}{*}{$\begin{array}{l}\text { Non-starch poly- } \\
\text { saccharides (NSP) of } \\
\text { different plant leaves. }\end{array}$} & \multirow{2}{*}{$\begin{array}{l}\text { Dry Weight } \\
\qquad \mathbf{g} / \mathbf{k g}\end{array}$} & \multirow{2}{*}{$\begin{array}{l}\text { Cellulose } \\
\text { g/kg }\end{array}$} & \multicolumn{7}{|c|}{ Non-celluloslc polysaccharldes (NCP), g/kg } \\
\hline & & & Rhamnose & Arablnose & Xylose & Mannose & Galactose & Glucose & Uronlc acld \\
\hline \multicolumn{10}{|l|}{ Turnip } \\
\hline soluble NSP & $192 \pm 16.4$ & 0,00 & $8 \pm 0.8$ & $12 \pm 0.84$ & $23 \pm 1.95$ & $22 \pm 2.1$ & $9 \pm 1.04$ & $56 \pm 3.2$ & $62 \pm 4.4$ \\
\hline insoduble NSP & $206 \pm 18.6$ & $108 \pm 5.6$ & $4 \pm 0.22$ & $2 \pm 0.02$ & $44 \pm 3.6$ & $11 \pm 0.9$ & $4 \pm 0.12$ & $13 \pm 1.1$ & $20 \pm 1.8$ \\
\hline Total NSP & $398 \pm 24.5$ & $108 \pm 6.6$ & $12 \pm 1.02$ & $14 \pm 1.04$ & $67 \pm 6.32$ & $33 \pm 2.4$ & $13 \pm 2.8$ & $69 \pm 3.9$ & $82 \pm 2.8$ \\
\hline \multicolumn{10}{|l|}{ Sugar beet } \\
\hline soluble NSP & $179 \pm 13.64$ & 0,00 & $13 \pm 1.10$ & $17 \pm 1.2$ & $22 \pm 2.4$ & $21 \pm 2.2$ & $2 \pm 0.06$ & $28 \pm 2.4$ & $76 \pm 6.2$ \\
\hline Insoduble NSP & $357 \pm 24.22$ & $102 \pm 7.2$ & $24 \pm 1.40$ & $45 \pm 1.32$ & $38 \pm 3.8$ & $68 \pm 46$ & $27 \pm 2.4$ & $25.6 \pm 5.1$ & $27.4 \pm 1.62$ \\
\hline Total NSP & $536 \pm 36.1$ & $102 \pm 8.2$ & $37 \pm 2.60$ & $62 \pm 3.8$ & $60 \pm 5.8$ & $89 \pm 5.5$ & $29 \pm 3.2$ & $53.6 \pm 3.8$ & $103.4 \pm 8.1$ \\
\hline \multicolumn{10}{|l|}{ Cabbrage } \\
\hline soluble NSP & $126 \pm 10.0$ & 0,00 & $2 \pm 0.04$ & $12 \pm 1.6$ & $11 \pm 0.96$ & $15.5 \pm 1.04$ & $3.5 \pm .94$ & $2 \pm 0.04$ & $80 \pm 4.6$ \\
\hline insoluble NSP & $352 \pm 18.3$ & $90 \pm 3.4$ & $24 \pm 1.02$ & $45 \pm 4.5$ & $38 \pm 3.4$ & $68 \pm 3.4$ & $27 \pm 1.04$ & $3 \mathbf{4} \pm 1.5$ & $26 \pm 2.2$ \\
\hline Total NSP & $478 \pm 29.2$ & $90 \pm 6.4$ & $26 \pm 1.4$ & $57 \pm 4.8$ & $49 \pm 2.6$ & $83.5 \pm 2.8$ & $30.5 \pm 3.1$ & $36 \pm 1.96$ & $106 \pm 8.1$ \\
\hline \multicolumn{10}{|l|}{ Fenugreek green } \\
\hline soluble NSP & $142 \pm 11.4$ & 0,00 & $4 \pm 0.36$ & $14.3 \pm 0.9$ & $6 \pm 0.34$ & $11 \pm \mathbf{0 . 8 4}$ & $0.7 \pm 0.02$ & $22 \pm 1.6$ & $84 \pm 5.6$ \\
\hline insoluble NSP & $526 \pm 34.3$ & $112 \pm 4.4$ & $46 \pm 2.80$ & $65 \pm 3.8$ & $45 \pm 3.2$ & $41 \pm 3.8$ & $32 \pm 2.2$ & $156 \pm 18.9$ & $29 \pm 3.5$ \\
\hline Total NSP & $668 \pm 38.8$ & $112 \pm 6.4$ & $50 \pm 3.40$ & $79.3 \pm 4.1$ & $51 \pm 4.1$ & $52 \pm 3.4$ & $32.7 \pm 2.8$ & $178 \pm 16.9$ & $113 \pm 7.4$ \\
\hline
\end{tabular}

Means of three batches (mean $\pm \mathrm{SE}$ )

Table II

Composition of the control and experimental diets

\begin{tabular}{lccccc}
\hline $\begin{array}{l}\text { Components } \\
\text { g/Kg diet }\end{array}$ & $\begin{array}{c}\text { control } \\
\text { diet }\end{array}$ & $\begin{array}{c}\text { Turnip } \\
\text { diet }\end{array}$ & $\begin{array}{c}\text { Sugar beet } \\
\text { diet }\end{array}$ & $\begin{array}{c}\text { Cabbage } \\
\text { diet }\end{array}$ & $\begin{array}{c}\text { Fenugreek } \\
\text { green diet }\end{array}$ \\
\hline Starch & 360,00 & 260,00 & 260,00 & 260,00 & 260,00 \\
Sucrose & 300,00 & 300,00 & 300,00 & 300,00 & 300,00 \\
Casein & 200,00 & 200,00 & 200,00 & 200,00 & 200,00 \\
Maize oil & 80,00 & 80,00 & 80,00 & 80,00 & 80,00 \\
Mineral mixture ${ }^{\text {b }}$ & 40,00 & 40,00 & 40,00 & 40,00 & 40,00 \\
Vitamine mixture ${ }^{\text {b }}$ & 20,00 & 20,00 & 20,00 & 20,00 & 20,00 \\
Dietary fibre & $-100,00$ & 100,00 & 100,00 & 100,00 \\
\hline
\end{tabular}

N.B. $100 \mathrm{~g}$ dietary fibres in tested samples are replaced for $100 \mathrm{~g}$ starch in the

a Johnson and Gee (1986)

b Jwanny et al. (1996)

amounts of soluble and insoluble NSP materials, while in sugar beet fibre the insoluble fraction was double the soluble one. The amount of insoluble fibre of cabbage was nearly three times than that of soluble NSP. Fenugreek green fibre contained 4 times more insoluble fibre than the soluble ones. In the soluble fibre fraction, higher levels of uronic acid were present $(62-84 \mathrm{~g} / \mathrm{kg})$ in the 4 plants than that insoluble one $(20-29 \mathrm{~g} / \mathrm{kg})$. Paper chromatographic analysis revealed that there were different values of individual monosaccharides of the non-cellulosic polysaccharides (NCP) in the 4 plant fibres (Table I).

\subsection{Diets and nutritional evaluation}

The diets were incorporated in the form of powder and they were well accepted, as the rats remained without any ill-health, but large faecal mass was 
Table III

Initial body weight, weight gain, PER, FER, Dapp and FCE of rats given control and experimental diets (Values are means with their standard errors for 7 animal / each 2 week / group)

\begin{tabular}{|c|c|c|c|c|c|c|c|c|c|c|c|c|}
\hline \multirow{2}{*}{$\begin{array}{r}\text { Diets } \\
\text { Parameters }\end{array}$} & \multirow{2}{*}{$\begin{array}{c}\text { Tlme } \\
\text { in } \\
\text { weeks }\end{array}$} & \multicolumn{2}{|c|}{$\begin{array}{l}\text { Control dliet } \\
\text { group }\end{array}$} & \multicolumn{3}{|c|}{ Turnip diet group } & \multicolumn{2}{|c|}{$\begin{array}{l}\text { Sugar beet } \\
\text { dlet group }\end{array}$} & \multicolumn{2}{|c|}{$\begin{array}{l}\text { Cabbage diet } \\
\text { group }\end{array}$} & \multicolumn{2}{|c|}{$\begin{array}{l}\text { Fenugreek green } \\
\text { dlet group }\end{array}$} \\
\hline & & Mean & \pm SE & Mean & \pm & SE & Mean & \pm SE & Mean & \pm SE & Mean & \pm SE \\
\hline \multirow{4}{*}{$\begin{array}{l}\text { Initial body } \\
\text { weight }(\mathbf{g})\end{array}$} & 2 & 115,00 & $\pm 0,68$ & 116,53 & \pm & 0,60 & 115,07 & $\pm 0,58$ & 114,82 & $\pm 0,17$ & 115,12 & $\pm 0,82$ \\
\hline & 4 & 113,62 & $\pm 0,73$ & 114,86 & \pm & 1,16 & $115,52^{a}$ & $\pm 0,60$ & $115,95^{a}$ & $\pm 0,81$ & 116,24 & $\pm 0,81$ \\
\hline & 6 & 115,64 & $\pm 0,79$ & 116,07 & \pm & 0,35 & 115,93 & $\pm 0,82$ & 114,14 & $\pm 1,20$ & 115,93 & $\pm 1,17$ \\
\hline & 8 & 115,14 & $\pm 1,35$ & 117,86 & \pm & 1,63 & 115,29 & $\pm 0,92$ & 115,71 & $\pm 1,27$ & 115,43 & $\pm 1,41$ \\
\hline \multirow{4}{*}{$\begin{array}{l}\text { Weight gain } \\
\text { (g) }\end{array}$} & 2 & 22,00 & $\pm 0,60$ & $15,54^{b}$ & \pm & 0,55 & $17,61^{b}$ & $\pm 0,46$ & $24,54^{a}$ & $\pm 0,86$ & $28,25^{b}$ & $\pm 0,86$ \\
\hline & 4 & 45,67 & $\pm 1,25$ & $29,52^{b}$ & \pm & 0,75 & $31,14^{b}$ & $\pm 1,02$ & 45,42 & $\pm 1,08$ & 45,33 & $\pm 1,67$ \\
\hline & 6 & 68,93 & $\pm 1,77$ & $43,21^{b}$ & \pm & 0,74 & $45,86^{b}$ & $\pm 1,64$ & $62,86^{a}$ & $\pm 1,51$ & $63,07^{a}$ & $\pm 1,88$ \\
\hline & 8 & 89,29 & $\pm 2,87$ & $50,00^{b}$ & \pm & 0,85 & $55,43^{b}$ & $\pm 2,34$ & $72,14^{b}$ & $\pm 1,28$ & $67,14^{b}$ & $\pm 1,90$ \\
\hline \multirow{4}{*}{$\begin{array}{l}\text { Food intake } \\
\text { (g) }\end{array}$} & 2 & 78,49 & $\pm 2,17$ & $58,73^{b}$ & \pm & 2,33 & $62,42^{b}$ & $\pm 1,55$ & $87,6^{b}$ & $\pm 0,54$ & $89,26^{a}$ & $\pm 3,20$ \\
\hline & 4 & 157,96 & $\pm 4,55$ & $118,28^{b}$ & \pm & 4,22 & $122,45^{b}$ & $\pm 5,15$ & 156,66 & $\pm 3,92$ & 152,61 & $\pm 3,62$ \\
\hline & 6 & 241,04 & $\pm 7,06$ & $187,64^{b}$ & \pm & 6,00 & $166,37^{b}$ & $\pm 5,81$ & $218,66^{a}$ & $\pm 5,82$ & $222,64^{a}$ & $\pm 5,77$ \\
\hline & 8 & 316,59 & $\pm 14,26$ & $202,97^{b}$ & \pm & 6,68 & $196,01^{\mathrm{b}}$ & $\pm 8,05$ & $245,28^{b}$ & $\pm 4,96$ & $227,48^{\mathrm{b}}$ & $\pm 8,33$ \\
\hline \multirow{4}{*}{ PER } & 2 & 2,31 & $\pm 0,01$ & $2,27^{b}$ & \pm & 0,01 & 2,30 & $\pm 0,02$ & $2,26^{\mathrm{b}}$ & $\pm 0,01$ & $2,33^{\mathrm{a}}$ & $\pm 0,01$ \\
\hline & 4 & 2,29 & $\pm 0,01$ & $2,29^{b}$ & \pm & 0,02 & 2,28 & $\pm 0,004$ & $2,31^{a}$ & $\pm 0,009$ & $2,31^{\mathfrak{a}}$ & $\pm 0,01$ \\
\hline & 6 & 2,30 & $\pm 0,01$ & $2,26^{b}$ & & 0,006 & $2,27^{\mathrm{b}}$ & $\pm 0,01$ & 2,29 & $\pm 0,009$ & 2,31 & $\pm 0,01$ \\
\hline & 8 & 2,30 & $\pm 0,01$ & $2,25^{b}$ & \pm & 0,02 & 2,29 & $\pm 0,01$ & 2,30 & $\pm 0,02$ & 2,30 & $\pm 0,02$ \\
\hline \multirow{4}{*}{ FER } & 2 & 0,28 & $\pm 0,003$ & $0,26^{b}$ & & 0,005 & 0,28 & $\pm 0,002$ & 0,28 & $\pm 0,003$ & 0,28 & $\pm 0,003$ \\
\hline & 4 & 0,29 & $\pm 0,002$ & $0,25^{\mathrm{b}}$ & & 0,004 & $0,26^{b}$ & $\pm 0,004$ & 0,29 & $\pm 0,002$ & 0,29 & $\pm 0,002$ \\
\hline & 6 & 0,29 & $\pm 0,002$ & $0,23^{b}$ & & 0,005 & $0,28^{a}$ & $\pm 0,003$ & 0,29 & $\pm 0,002$ & 0,29 & $\pm 0,002$ \\
\hline & 8 & 0,28 & $\pm 0,010$ & $0,25^{b}$ & \pm & 0,01 & 0,28 & $\pm 0,004$ & 0,29 & $\pm 0,003$ & $0,30^{a}$ & $\pm 0,003$ \\
\hline \multirow{4}{*}{ Dapp } & 2 & 92,51 & $\pm 0,30$ & $87,7^{a}$ & \pm & 0,45 & $88,39^{b}$ & $\pm 0,61$ & $90,53^{a}$ & $\pm 0,28$ & 92,42 & $\pm 0,24$ \\
\hline & 4 & 92,77 & $\pm 0,22$ & $83,31^{\mathrm{b}}$ & \pm & 0,48 & $90,21^{b}$ & $\pm 0,30$ & $91,99^{\mathrm{a}}$ & $\pm 0,19$ & 92,16 & $\pm 0,23$ \\
\hline & 6 & 92,99 & $\pm 0,30$ & $81,79^{b}$ & \pm & 0,41 & $90,59^{b}$ & $\pm 0,30$ & $92,24^{a}$ & $\pm 0,22$ & 93,15 & $\pm 0,26$ \\
\hline & 8 & 93,79 & $\pm 0,28$ & $89,04^{b}$ & \pm & 0,38 & $91,79^{b}$ & $\pm 0,30$ & $93,31^{\mathbf{a}}$ & $\pm 0,19$ & 93,89 & $\pm 0,07$ \\
\hline \multirow{4}{*}{ FCE } & 2 & 29,64 & $\pm 0,40$ & $26,32^{b}$ & \pm & 0,47 & $28,18^{\text {a }}$ & $\pm 0,28$ & $28,14^{\mathrm{a}}$ & $\pm 0,31$ & $28,5^{\text {a }}$ & $\pm 0,23$ \\
\hline & 4 & 28,90 & $\pm 0,21$ & $25,14^{b}$ & \pm & 0,33 & $25,67^{b}$ & $\pm 0,38$ & 28,90 & $\pm 0,18$ & 28,90 & $\pm 0,22$ \\
\hline & 6 & 28,64 & $\pm 0,23$ & $23,21^{b}$ & \pm & 0,52 & $27,57^{\mathrm{a}}$ & $\pm 0,31$ & 28,79 & $\pm 0,24$ & 28,43 & $\pm 0,25$ \\
\hline & 8 & 28,43 & $\pm 1,11$ & $22,86^{b}$ & \pm & 1,14 & 28,29 & $\pm 0,36$ & 29,43 & $\pm 0,30$ & 29,57 & $\pm 0,30$ \\
\hline
\end{tabular}

a Significant $(p<0,05)$ within row

b Significant $(p<0,01)$ within row

present. The results in table III showed that, at the end of feeding period, the weight gain and food intake of the rats fed on the 4 experimental diets have highly significant decrease than those rats fed the control diet. Generally, rats fed turnip diet for 2-8 weeks exhibited a highly significant decrease in all the nutritional parameters, while rats fed sugar beet fiber-containing diet a highly significant decrease was observed only in the value of weight gain, food intake and Dapp through the feeding period comparing with the control. No difference was observed in the value of PER FER, Dapp and FCE 
Table IV

Total lipids, total cholesterol, HDL-C, LDL-C and triglycerides levels in serum of rats fed on control and experimental diets (Values are means with their standard errors for 7 animal / each 2 week / group)

\begin{tabular}{|c|c|c|c|c|c|c|c|c|c|c|c|c|c|c|c|c|}
\hline \multirow[b]{2}{*}{ Parameters } & \multirow{2}{*}{$\begin{array}{l}\text { Tlme } \\
\text { in } \\
\text { weeks }\end{array}$} & \multicolumn{3}{|c|}{$\begin{array}{l}\text { Control diet } \\
\text { group }\end{array}$} & \multicolumn{3}{|c|}{ Turnip diet group } & \multicolumn{3}{|c|}{$\begin{array}{l}\text { Sugar beet } \\
\text { dlet group }\end{array}$} & \multicolumn{3}{|c|}{$\begin{array}{c}\text { Cabbage dlet } \\
\text { group }\end{array}$} & \multicolumn{3}{|c|}{$\begin{array}{l}\text { Fenugreek green } \\
\text { dlet group }\end{array}$} \\
\hline & & Mean & \pm & SE & Mean & \pm & SE & Mean & \pm & SE & Mean & \pm & SE & Mean & \pm & SE \\
\hline \multirow{4}{*}{$\begin{array}{l}\text { Total lipidts } \\
\text { (mg\%) }\end{array}$} & 2 & 455,60 & \pm & 4,58 & 454,40 & \pm & 3,82 & $405,32^{b}$ & \pm & 2,31 & $382,14^{b}$ & \pm & 3,05 & $368,44^{b}$ & \pm & 3,46 \\
\hline & 4 & 467,5 & \pm & 3,07 & 461,90 & \pm & 5,32 & $367,42^{b}$ & \pm & 3,07 & $360,10^{b}$ & \pm & 2,30 & $344,90^{b}$ & \pm & 3,83 \\
\hline & 6 & 468,9 & \pm & 2,66 & $475,10^{a}$ & \pm & 6,10 & $336,56^{b}$ & \pm & 3,79 & $312,46^{b}$ & \pm & 3,45 & $294,16^{b}$ & \pm & 3,04 \\
\hline & 8 & 476,30 & \pm & 3,43 & $486,64^{b}$ & \pm & 4,57 & $329,94^{b}$ & \pm & 5,31 & $280,66^{b}$ & \pm & 2,67 & $275,10^{b}$ & \pm & 4,57 \\
\hline \multirow{4}{*}{$\begin{array}{l}\text { Total } \\
\text { cholesterol } \\
(\mathrm{mg} \%)\end{array}$} & 2 & 133,4 & \pm & 1,57 & 130,24 & \pm & 1,20 & $122,20^{b}$ & \pm & 1,15 & $110,34^{b}$ & \pm & 1,57 & $112,96^{b}$ & \pm & 2,32 \\
\hline & 4 & 130,3 & \pm & 1,97 & 127,92 & \pm & 1,55 & $109,66^{b}$ & \pm & 1,61 & $96,26^{\mathrm{b}}$ & \pm & 2,28 & $96,24^{b}$ & \pm & 1,55 \\
\hline & 6 & 125,70 & \pm & 0,81 & 126,40 & \pm & 3,50 & $102,82^{B}$ & \pm & 2,04 & $81,44^{b}$ & \pm & 1,19 & $87,66^{b}$ & \pm & 1,96 \\
\hline & 8 & 128,40 & \pm & 1,15 & 125,80 & \pm & 3,05 & $97,26^{b}$ & \pm & 2,31 & $80,16^{b}$ & \pm & 0,73 & $80,89^{b}$ & \pm & 3,17 \\
\hline \multirow{4}{*}{ HDL-C (mg\%) } & 2 & 26,24 & \pm & 0,76 & 25,40 & \pm & 2,68 & 25,6 & \pm & 0,77 & 26,82 & \pm & 1,52 & 27,90 & \pm & 1,52 \\
\hline & 4 & 28,86 & \pm & 1,24 & 26,840 & \pm & 3,41 & 27,90 & \pm & 1,52 & 29,88 & \pm & 1,15 & 30,74 & \pm & 2,41 \\
\hline & 6 & 27,22 & \pm & 1,52 & 25,240 & \pm & 3,99 & 26,24 & \pm & 1,14 & 28,72 & \pm & 2,28 & 29,40 & \pm & 2,66 \\
\hline & 8 & 25,96 & \pm & 1,90 & 23,44 & \pm & 3,04 & 24,96 & \pm & 3,17 & 27,72 & \pm & 3,04 & 28,34 & \pm & 3,41 \\
\hline \multirow{4}{*}{$\begin{array}{l}\text { LDL-C } \\
\text { (mg\%) }\end{array}$} & 2 & 64,52 & \pm & 2,27 & 25,90 & \pm & 2,72 & $55,92^{a}$ & \pm & 2,38 & $48,94^{b}$ & 王 & 2,34 & $44,98^{b}$ & \pm & 1,17 \\
\hline & 4 & 67,12 & \pm & 1,52 & 60,10 & \pm & 3,86 & $60,10^{7}$ & \pm & 3,10 & $38,20^{b}$ & \pm & 4,46 & $40,64^{b}$ & \pm & 0,98 \\
\hline & 6 & 64,10 & \pm & 3,41 & 62,46 & \pm & 3,48 & 56,17 & \pm & 3,55 & $32,44^{b}$ & \pm & 3,52 & $34,70^{b}$ & \pm & 0,91 \\
\hline & 8 & 66,26 & \pm & 1,15 & 62,38 & \pm & 3,10 & $58,10^{2}$ & \pm & 3,82 & $36,99^{b}$ & \pm & 3,82 & $36,16^{b}$ & \pm & 1,36 \\
\hline \multirow{4}{*}{$\begin{array}{l}\text { Triglycerides } \\
(\mathrm{mg} \%)\end{array}$} & 2 & 187,10 & $\mathbf{\pm}$ & 1,25 & $176,26^{b}$ & \pm & 2,41 & $162,32^{b}$ & \pm & 1,15 & $168,36^{b}$ & \pm & 0,77 & $160,92^{b}$ & \pm & 1,60 \\
\hline & 4 & 196,3 & \pm & 1,65 & $173,90^{b}$ & \pm & 1,56 & $150,42^{b}$ & \pm & 0,79 & $141,30^{b}$ & $I$ & 1,14 & $152,32^{b}$ & \pm & 1,95 \\
\hline & 6 & 198,20 & $I$ & 2,35 & $170,48^{b}$ & \pm & 1,93 & $137,12^{b}$ & \pm & 1,86 & $130,92^{b}$ & \pm & 1,55 & $127,60^{b}$ & \pm & 1,16 \\
\hline & 8 & 196,90 & \pm & 1,90 & $169,94^{b}$ & \pm & 1,19 & $132,24^{b}$ & \pm & 2,27 & $134,48^{b}$ & \pm & 0,76 & $122,26^{b}$ & \pm & 2,37 \\
\hline
\end{tabular}

a Significant $(p<0,05)$ within row b Significant $(\mathrm{p}<0,01)$ within row

when rats received cabbage and fenugreek green fibre-containing diets (Table III), compared with rats fed control diet .

\subsection{Diets and serum lipid parameters}

Plasma lipid responses of the rats fed the control and the experimental diets are given in Table IV. The group received control diet, showed significant increase in total lipid and triglycerides levels with increasing the feeding period, particularly at 6,8 weeks feeding. The rats fed sugar beet, cabbage and fenugreek green fibre-containing diets, had highly significant decrease in the levels of serum total lipid, total cholesterol, LDL-C (except sugar beet) and triglycerides, comparing with those received the control diet throughout the 8 weeks feeding. The turnip fibre-containing diet had only highly significant decrease on the level of serum triglycerides over the feeding period. No significant change was observed in serum HDL-C level within all the 4 experimental diets.

\subsection{Diets and liver lipid parameters}

The present results (Table V) show that total lipid and total cholesterol levels in the liver of rats given cabbage and fenugreek green fibre-containing diet, are highly significant decrease than those received control diet over the feeding period. While highly significant decrease was detected in triglycerides levels in liver of rats fed cabbage (after 2 weeks feeding only) and fenugreek green fibre-containing diets, compared to those fed control and other two experimental diets, over the feeding period. 
Table V

Total lipids, total cholesterol and triglycerides levels in liver of rats given control and experimental diets (Values are means with their standard errors for 7 animal / each 2 week / group)

\begin{tabular}{|c|c|c|c|c|c|c|c|c|c|c|c|c|c|c|c|c|}
\hline \multirow[b]{2}{*}{ Parameters } & \multirow{2}{*}{$\begin{array}{c}\text { Time } \\
\text { in } \\
\text { weeks }\end{array}$} & \multicolumn{3}{|c|}{ Control diet group } & \multicolumn{3}{|c|}{ Turnip diet group } & \multicolumn{3}{|c|}{$\begin{array}{l}\text { Sugar beet } \\
\text { dlet group }\end{array}$} & \multicolumn{3}{|c|}{$\begin{array}{c}\text { Cabbage dlet } \\
\text { group }\end{array}$} & \multicolumn{3}{|c|}{$\begin{array}{l}\text { Fenugreek green } \\
\text { dlet group }\end{array}$} \\
\hline & & Mean & \pm & SE & Mean & \pm & SE & Mean & \pm & SE & Mean & \pm & SE & Mean & \pm & SE \\
\hline \multirow{4}{*}{$\begin{array}{l}\text { Total lipiids } \\
(\mathrm{mg} / \mathrm{g})\end{array}$} & 2 & 14,72 & \pm & 0,44 & 14,66 & \pm & 0,65 & 14,46 & \pm & 0,32 & $11,40^{b}$ & \pm & 0,49 & $9,14^{b}$ & \pm & 0,38 \\
\hline & 4 & 14,45 & \pm & 0,63 & 14,04 & \pm & 0,57 & 13,84 & 未 & 0,26 & $9,63^{b}$ & 未 & 0,44 & $7,96^{\mathrm{b}}$ & \pm & 0,27 \\
\hline & 6 & 13,94 & \pm & 0,70 & 12,90 & \pm & 0,65 & $11,64^{b}$ & \pm & 0,40 & $8,92^{b}$ & \pm & 0,18 & $7,90^{b}$ & \pm & 0,31 \\
\hline & 8 & 14,86 & \pm & 0,46 & $13,16^{\mathrm{a}}$ & \pm & 0,59 & $11,31^{b}$ & \pm & 0,35 & $8,72^{b}$ & \pm & 0,25 & $8,62^{\mathrm{b}}$ & \pm & 0,74 \\
\hline \multirow{4}{*}{$\begin{array}{l}\text { Total } \\
\text { cholesterol } \\
(\mathrm{mg} / \mathrm{g})\end{array}$} & 2 & 1,36 & \pm & 0,17 & 1,36 & \pm & 0,04 & 1,17 & \pm & 0,06 & $0,82^{b}$ & \pm & 0,07 & $0,67^{b}$ & \pm & 0,05 \\
\hline & 4 & 1,42 & \pm & 0,23 & 1,32 & \pm & 0,03 & 1,06 & \pm & 0,08 & $0,74^{\mathrm{b}}$ & \pm & 0,06 & $0,60^{b}$ & \pm & 0,06 \\
\hline & 6 & 1,31 & \pm & 0,13 & 1,22 & \pm & 0,05 & $0,94^{a}$ & \pm & 0,05 & $0,66^{b}$ & \pm & 0,05 & $0,58^{b}$ & \pm & 0,04 \\
\hline & 8 & 1,45 & \pm & 0,18 & 1,38 & \pm & 0,08 & $0,89^{a}$ & \pm & 0,10 & $0,52^{\mathrm{b}}$ & \pm & 0,03 & $0,50^{\mathrm{b}}$ & \pm & 0,15 \\
\hline \multirow{4}{*}{$\begin{array}{l}\text { Triglycerides } \\
(\mathrm{mg} / \mathrm{g})\end{array}$} & 2 & 4,41 & \pm & 0,25 & 3,91 & \pm & 0,33 & 4,28 & \pm & 0,44 & $2,83^{b}$ & \pm & 0,20 & $2,32^{b}$ & \pm & 0,15 \\
\hline & 4 & 4,30 & \pm & 0,40 & 3,93 & \pm & 0,32 & 4,10 & \pm & 0,25 & $3,16^{a}$ & \pm & 0,12 & $1,86^{b}$ & \pm & 0,11 \\
\hline & 6 & 3,97 & \pm & 0,21 & 3,76 & \pm & 0,16 & 3,69 & \pm & 0,16 & $2,89^{a}$ & \pm & 0,34 & $1,84^{\mathrm{b}}$ & \pm & 0,20 \\
\hline & 8 & 3,93 & \pm & 0,38 & 3,89 & \pm & 0,27 & 3,57 & \pm & 0,17 & $2,71^{a}$ & \pm & 0,39 & $1,71^{\mathrm{b}}$ & \pm & 0,09 \\
\hline
\end{tabular}

a Significant $(p<0,05)$ within row

b Significant $(p<0,01)$ within row

\section{DISCUSSION}

Lajvardi et al., (1993) and Campbell et al., (1997) demonstrated that the different effects of dietary fibres are dependant on their type (dose, structure, soluble and insoluble NSP) and on the duration of the experiment. Madar, (1990) and Younes et al., (2001) found that dietary fibres of vegetable products may affect the bioavailability impairment of mineral absorption, is traditionally ascribed to the uronic acid content of fibre fraction. The present study includes different types of natural fibre sources, that are available for human consumption, which can be divided into 4 groups with respect to their solubility percentages (Table I). The results show that, the differences were not only in the ratio between soluble and insoluble NSP in the four plant leaves but also in their monosaccharide constituents. The uronic acid content of soluble fibres were higher than of the insoluble one Similar results were obtained by other investigators by using cabbage, sugar beet, Jerusalem artichoke and rhubarb (Roland et al., 1995; Goel et al., 1997, Bennami-Kabochi et al., 2000 and Guerin et al., 2001). These results are inconsistent to other studies made by Anderson et al., (1990) and Topping, (1991). Johnson and Gee, (1986), Vahouny, (1987), Galibois et al., (1994), Roland et al., (1995) and Jwanny et al., (1996) have previously suggested that dietary fibres of guar gum, wheat bran, alfalfa, date waste and cellulose, had lowering effect on nutritional parameter (food intake, FER, Dapp and
FCE) and growth rate of rats. In line with these observations the nutritional parameters-lowering effect of rats fed turnip fibre supplemented diet was also evident in the present study. Significant lower in food intake, FER, Dapp and FCE values in rats received both sugar beet and turnip fibre-containing diets, over the feeding period, compared to that given control diet. Kritchevsky et al., (1988), Goodlad and Mathers (1990) and Roland et al., (1995), found that these differences are related to the presence of fibres sources in the diets at the level of $100 \mathrm{~g} / \mathrm{kg}$ diet. The values of PER for rats given the 4 experimental diets were higher than those mentioned by Bach-Knudsen et al., (1994), using Jerusalem artichoke fibre.

Ebihara and Schneeman, (1989), Galibois et al., (1994), Jwanny et al., (1996) and Terpstra et al., (2000) has been strongly suggested that the consumption of food rich in dietary fibre or supplements of the purified components, could be beneficial in terms of reducing hypercholesterolemic, aortic atherosclerosis, hyperlipidemic and deposition of tissue cholesterol in human and animals. The present results clearly showed that a combination of different carbohydrate (soluble and insoluble NSP), in different levels, has synergistic effect on lipid metabolism (Younes et al., 2001). Both animal and clinical studies have previously suggested that dietary fibre (psyillum and rhubarb) can be potentially a hypocholesterolemic agent (Basu et al., 1993; Goel et al., 1997 and Terpstra et al., 2000). 
Consistent with these finding, the cholesterollowering effect of the fibre sources of sugar beet, cabbage and fenugreek green plant was cleared in the present study. Highly significant decrease in the level of serum triglycerides of rats fed the 4 experimental diets through the feeding period was observed (table IV). These finding are in accordance with other studies of Anderson et al., (1990), Topping, (1991), Goel et al., (1999) and Gallaher et al., (2000). Dodson et al., (1981), Madar and Stark, (1995) and Gallaher et al., (2000) stated that positive correlation exists between the incidence of coronary atherosclerosis and plasma LDL-C concentration, which act as cardiovascular risk factor. So higher reduction in LDL-C levels in sera of rats fed cabbage, fenugreek green, sugar beet and turnip fibre-containing diets $(49 \%, 45 \%, 13 \%$ and $10 \%$ respectively), means that these fibre sources can have an effect on lowering the incidence of coronary atherosclerosis and reducing in risk factors for cardiovascular diseases. These findings are in accordance with other investigations (Ryden and Robertson, 1995 and Buhman et al., 1998).

Also, rats fed cabbage and fenugreek green diets showed significant reduction in hepatic total lipid and cholesterol levels over the feeding periods compared to those fed control diet. Higher reduction in hepatic triglyceride levels was observed in rats given fenugreek green fiber-containing diet $(56 \%)$, whereas in other studies a reduction of up to $33 \%$ was achieved (Jwanny et al., 1996 and Bennami-Kabochi et al., 2000). The results of the present study were in accordance to those reported by Buhman et al., (1998) and Gallaher et al., (2000), but contradict to Kritchevsky et al., (1988) and Langkidle et al., (1993), who indicated that alfalfa fibre supplemented to the diet elevated the levels of these hepatic lipid components.

\section{CONCLUSION}

Accordingly it can be concluded that the use of each of fenugreek green, cabbage, sugar beet dietary fibres in the diet $(100 \mathrm{~g} / \mathrm{kg}$ diet) have a great loss in lipid components of serum and liver of rats and could used as a protective factor against atherosclerosis, coronary heart diseases.

\section{REFERENCES}

Anderson, J. W, Deakins, D. A., Floore, T. L., Smith, B.M Whitis, S. E., 1990. Dietary fibre and coronary heart disease. Crit. Revs. in Food Sci. and Nutr. 29, 95-136.

Bach-Knudsen, K. E., Wisker, E., Daniel M., Feldhein, W., Eggum, B.O., 1994. Digestibility of energy, protein, fat and non-starch polysaccharides in mixed diets: Comparative studies between man and rat. Br. J. Nutr. 71, 471-487.

Basu, T. K., Ooraikul, Garg, M., 1993. The lipid lowering effects of rhubarb stalk fibre: anew source of dietary fibre. Nutr. Res. 13, 1017-1024.
Bennami-Kabochi, N., Fdhil, H., Cherrah, Y. Bouayadi, F.E.L., Kohel, L., Marquie, G., 2000. Therapeutic effect Olea europea var. Oleaster leaves on lipid and carbohydrate metabolism in obese and prediabetic sand rats (Psammomys obesus). Ann. Pharm. Fr. 58, sand rats

Birt, D. F., Markin, R. S., Blackwood, D., Harvell, D. M. E., Shuel, J. D., Pennington, K.L., 1998. Dietary lignin, an insoluble fibre, enhanced uterine cancer but did not influence mammary cancer induced by $\mathrm{N}$-methyl-N-nitrosourea in rats. Nutr. Cancer. Mahwah N.J.: Lawrence Erlbaum Associates, Inc. 31, 24-30.

Buhman, K.K., Furumoto, E.J., Donkin, S. S., Story, J. A., 1998. Dietary Psyllium increases fecal bile acid excretion, total steroid excretion and bile acid biosynthesis in rats. J. Nutr. 128, 1199-1203.

Campbell, J.M., Fahey, G.C., Wolf B.W., 1997. Selected indigestible oligosaccharides affect large bowel mass, cecal and fecal short chain fatty acid, $\mathrm{pH}$ and cecal and fecal short chain fatty aci
microflora in rats. J. Nutr. 127, 130-136.

microflora in rats. J. Nutr. 127, $130-136$.
Dodson, P.M., Stocks, J., Holdsworth, G., Galton, D. J., 1981. High-fibre and low-fat diets in diabetes mellitus. J. Nutr. 46, 289-294.

Ebihara, K., Schneeman, B. O., 1989. Interaction of bile acids, phospholipids, cholesterol and triglycerides with dietary fibres in small intestine of rats. J. Nutr. dietary fibres in

Englyst, H.N., Cummings, J.H., 1988. Improved method for measurements of dietary fibre as non-starch polysaccharides in plant foods. J. Assoc. Off. Annal. Chem. 71, 808-814.

Fisher, R.A., 1970. Statistical method for research workers, Edinburg et. 14, Oliver and Boyd P. 140-142.

Folch, J., Lees, M., Sloane-Stanley G.H., 1957. A simple method for the isolation and purification of total lipids from animal tissue. J. Biol. Chem. 226, 497-509.

Galibois, I., Destosiers, T., Guevin, N., Lavigne, C., Jacques H., 1994. Effect of dietary fibre mixtures on glucose and lipid metabolism and on mineral absorption in the rat. Ann. Nutr. Metab. 88, 203-211.

Gallaher, C.M., Munion, J., Hesslink, R. J., Wise, J., Gallaher D. D., 2000. Cholesterol reduction by glucomannan and chitosan is mediated by changes in cholesterol absorption and bile acid and fat excretion in rats. J. Nutr. 130, 2753-2759.

Goel, V., Cheema, S. K., Agellon, L. B., Ooraikul, B., Basu, T.K., 1999. Dietary rhubarb, Rheum rhaponticum. stalk fibre stimulates cholesterol 7 a-hydroxylase gene expression and bile acid excretion in cholesterol fed $C$ 57BL/6 J mice. Br. J. Nutr. 81, 65-71.

Goel, V., Oorakiul, B., Basu T.K., 1997. Cholesterol Lowering effects of rhuborb fibre in hypercholesterolemic men. J. Am. Coll. Nutr. 16, 600-604

Goodlad, J. S., Mathers, J.C., 1990. Large bowel fermentation in rats given diets containing raw peas, Pisum sativum. Br. J. Nutr. 64, 569-587.

Guerin, S., Ramonet, Y., Le-Cloavec, J., Meunier-Salaun, M.C., Bourgeut, P., Malbert C.H., 2001. Changes in intragastric meal distribution are better predictors of gastric rate in conscious pigs than are meal viscosity or dietary fibre concentration. Br. J. Nutr. 85, 343-350.

Jacobs, R. 1983. Effect of short-term dietary fat on cell growth in rat gastrointestinal mucous and pancreas. growth in rat gastrointestinal

Johnson, I. T., Gee J.M., 1986. Gastrointestinal adaptation in response to soluble non-available polysaccharides in the rat. Br. J. Nutr. 55, 497-505.

Jwanny, E.W., Hussein M. M., 1976. Carbohydrates from hydrocarbons. II Free and bound sugars from yeast 
cells grown on $\mathrm{n}$-Hexadecane. Acta Biol. Acad. Sci. Hung. 27, 101-106.

Jwanny, E.W., Rashad M. M., Moharib, S. A., El-Beih, N M., 1996. Biological evaluation of data waste dietary fibre and Endomycopsis fibuligera protein with rats. Bioresource Technology. 56, 201-205.

Knight, J. A., Anderson, S., Rewale, J. M., 1972. Chemical basis of the sulfophosphovanillin reaction for estimating total serum lipids. Clin. Chem. 18, 199-202.

Kritchevsky, D., Tepper, S.A., Satchithanondem, S., Cassidy, M. M., Vahouny, G.V., 1988. Dietary fibre supplements. Effect on serum and liver lipids and on liver phospholipids composition in rats. Lipids 23 , liver phosph

Lajvardi, A., Mazarin, G.I., Gillespie B., Satchithanadam, S., Colvert, R.J., 1993. Starches of varied digestibilities modify intestinal function in rats. J. Nutr. 123, 2059-2066.

Langkidle, A.M., Andersson, H., Bosaeus, I., 1993. Sugar beet fibre increases cholesterol and reduces bile acid excretion from small bowel. Br. J. Nutr. 70,757-766.

Lopes-Virella, M.F., Stone, P., Ellis, S., Colwell J. A., 1977.Cholesterol determination in high-density lipoprotein separated by three different methods. Clin. Chem. 23, 582-584.

Madar, Z., 1990. Dietary fibre from legume seeds in control of blood lipids and glucose. Proceedings of the joint CEC-NCRO workshop held in Israel, Ginozor Kibbutz. Eur. Publication, 264, 115-133.

Madar, Z., Stark, A., 1995. Possible mechanisms by which dietary fibres affect lipid metabolism. Agro-Food-Industry Hi-Tech. 40-42.

Moharib, S.A., 2000. Studies on intestinal enzyme activity and nutritive values of dietary fibres in rats. Bull. Fac. Agric. Cairo Univ. 51, 431-446.

Nyman, M., Asp, N.G., 1985. Dietary fibre fermentation in the rat intestinal tract: effect of adaptation period, protein and fibre level and particle size. Br. J. Nutr. 54 , 635-643.

Onning, G., Asp, N.G., 1995. Effect of oat saponin and different types of dietary fibre on the digestion of carbohydrates. Br. J. Nutr. 74, 229-237.

Ranhorta, C. S., Voewe, R.J., Pyat, L.V., 1976. Effect of some wheat mill-fractions on blood and liver lipids in cholesterol fed rats. Cereal Chem. 50, 540-547.

Rashad, M.M., Moharib, S.A., Abdou, H.M., 2000. Chemical constituents and nutritive value of 6 local vegetable leaves byproducts. J. Agric. Sci. Mansura Univ. 25, 7229-7238.
Roland, N., Nugon-Bandon, L., Andieuz, C., Szylit, O., 1995. Comparative study on the fermentative characteristics of inulin and different types of fibre in rats inoculated with a human whole faecal flora. Br. J. Nutr. 74, 239-249.

Ryden, P., Robertson, J. A., 1995. The consequences of fruit and vegetable fibre fermentation on their binding capacity for Me-IQX and the effects of soluble fibre sources on the binding affinity of wheat bran preparations. Carcinogenesis. 8, 1711-1716.

Sapuntzakis, M. S., Bowen, P. E., Hussain, E.A., Damayanti-wood, B. I., Farnsworth N. R., 2001. Chemical composition and potential health effects of prunes: A functional food. Crit. Rev. Food. Sci. and Nutr. 41, 251-286

Silva, M.E.S.T., Nicoli, J.R., 1985. Production and nutritive value of SCP from Fusarium oxysporum Var. Lini grown. In vinasse. J. Ferment. Technol. 63, 41-49.

Terpstra, A.H.M., Lapre, J.A., de-Vries, H. T., Beynen, A. C., 2000. Hypocholesterolemic effect of dietary psyllium in female rats. Ann. Nutr. Metab. 44, 223-228.

Topping, D. L., 1991. Soluble fibre polysaccharides: Effect on plasma cholesterol and colonic fermentation. Nutr. Revs. 49, 195-203.

Trinder, P., 1969. Simple turbidimetric method for the determination of serum cholesterol. Ann. Clin. Biochem. 6, 165-166.

Vahouny, G.V., Kdalafi, R., Satchithanandam, S., Watkins, R. W., Story, J. A., Cassidy, M. A., Kritchevsky, D., 1987. Dietary fibre supplementation and faecal bile acids neutral steroids and divalent cations in rats. J. Nutr. 117, 2009-2015.

Wahlefeld, A.W. 1974. Triglycerides determination after enzymatic hydrolysis. In: HV Bergmeyer. ed. Methods enzymatic hydrolysis. In: HV Bergmeyer. ed. Methods $3^{\text {rd }}$ German ed.) p. 1831-1835. Verlag Chemie
. $3^{\text {rd }}$ German ed.) p. 1831-1835. Verlag Chemie
Weinheim and Academic Press, Inc. New York and London.

Wilson, C.M., 1959. Quantitative determination of sugars on paper chromatograms. Anal. Chem.31, 1199-1201.

Younes, H., Coudary, C., Bellanger, J., Demigne, C., Rayssiguier, Y., Remesy, C., 2001. Effect of two fermentable carbohydrates (inulin and resistant starch) and their combination on calcium and magnesium balance in rats. Br. J. Nutr. 86, 479-485.

Recibido: Septiembre 2002 Aceptado: Febrero 2003 